\title{
Realization of Enhanced Evanescent Field Long Period Fiber Grating Near Turn around Point for Label-Free Immunosensing ${ }^{\dagger}$
}

\author{
Tanoy Kumar Dey ${ }^{1}$, Sara Tombelli ${ }^{2}$, Palas Biswas ${ }^{1}$, Ambra Giannetti ${ }^{2}$, Nandini Basumallick ${ }^{1}$, \\ Francesco Baldini ${ }^{2}$, Somnath Bandyopadhyay 1,** and Cosimo Trono 2,* \\ 1 Fiber Optics \& Photonics Division, CSIR_Central Glass \& Ceramic Research Institute, 196 Raja S. C. \\ Mullick Road, Kolkata 700032, India; tanoykumardey@gmail.com (T.K.D.); palas@cgcri.res.in (P.B.); \\ nandini_b@cgcri.res.in (N.B.) \\ 2 Institute of Applied Physics “Nello Carrar", CNR-IFAC, Via Madonna del Piano 10, 50019 Sesto Fiorentino, \\ Italy; s.tombelli@ifac.cnr.it (S.T.); a.giannetti@ifac.cnr.it (A.G.); f.baldini@ifac.cnr.it (F.B.) \\ * Correspondence: somnath@cgcri.res.in (S.B.); c.trono@ifac.cnr.it (C.T.) \\ + Presented at the 1st International Electronic Conference on Biosensors, 2-17 November 2020; \\ Available online: https://iecb2020.sciforum.net/.
}

Received: date; Accepted: date; Published: date

\begin{abstract}
A Long period Fiber Grating (LPFG) with maximum enhancement of evanescent field has been designed and fabricated, along with theoretical modeling, by working near the turn-around point (TAP) of lowest order symmetric cladding mode ( $\mathrm{LP}_{0,2}$ cladding mode). The LPFG was fabricated using a point by point inscription technique and it was characterized in term of surrounding refractive index (SRI) within the range of 1.333 to 1.3335 using a thermostated flowcell. This closed cell, made of poly (methyl methacrylate) (PMMA), was designed for better handling of the sensor, because during the fabrication process the diameter of the LPFG was reduced up to $\sim 20 \mu \mathrm{m}$ by chemical etching, for the maximum enhancement of the evanescent field. The sensitivity of dual peak resonance of the $\mathrm{LP}_{0,2}$ cladding mode near TAP was measured and resulted to be $\sim 8751$ $\mathrm{nm} / \mathrm{SRIU}$ with a resolution of the order of $10^{-5} \mathrm{RIU}$. The sensor was further used for the label-free immunosensing application by the implementation of Immunoglobulin G (IgG)/ antiImmunoglobulin $\mathrm{G}$ (anti-IgG) bioassay in human serum on the grating region inside the thermostated closed flow cell.
\end{abstract}

Keywords: long period fiber grating; evanescent field; biosensor

\section{Introduction}

LPFGs are turning out to be promising bio-chemical sensors for their ability to sense surrounding refractive index (SRI) changes [1-4]. In most of the bio-chemical applications, SRI is $\sim 1.333$ [5-7], being the bioanalytes dissolved in aqueous solutions. However, the sensitivity of the sensor in this RI range is not very high [8]. Different methodologies have been developed by the researchers throughout the years to enhance the sensitivity of the sensor, such as operating around mode transition [5,9-12], operating near cladding modes (CMs) TAP [13,14], enhancement of evanescent field of CMs $[13,15,16]$. Combination of these methodologies has also been developed [1722]. Recently, it has been shown that by reducing the cladding diameter of a LPFG, a lower order CM (dispersed CM) can be obtained near TAP $[18,23]$. The dispersed lower order CM becomes more sensitive than the normal higher order CM near TAP due to the enhancement of the evanescent field $[18,23]$. In this work, enhancement of sensitivity of the LPFG is achieved by the combination of two methodologies: (1) maximum enhancement of the evanescent field and (2) working near the TAP of 
a CM. A LPFG is fabricated in $125 \mu \mathrm{m}$ single mode fiber and the cladding diameter is reduced up to $\sim 21 \mu \mathrm{m}$ to obtain the lowest order dispersed $\mathrm{CM}\left(\mathrm{LP}_{0,2}\right)$ so as to attain the maximum enhancement of the evanescent field and of the TAP of the $\mathrm{LP}_{0,2} \mathrm{CM}$. By theoretical modelling, grating period and refractive index modulation is optimised. A closed flow cell is fabricated to analyse the sensitivity of the sensor in order to avoid the problems associated to both fragility and difficult handling. The sensitivity is found out to be $8751 \mathrm{~nm} / \mathrm{SRIU}$ within the RI range 1.333-1.3335 for the dual peak resonance of $\mathrm{LP}_{0,2} \mathrm{CM}$. Moreover, a label free immunoassay using IgG/ anti-IgG interaction was performed using the sensor and the specificity of the sensor was confirmed by a negative control test using human serum.

\section{Materials and Methods}

\subsection{Materials}

Hydrofluoric acid (HF), sodium chloride $(\mathrm{NaCl})$, ethanol (EtOH), goat anti-mouse IgG, bovine serum albumin (BSA) and the reagents for phosphate buffer saline (PBS, $0.01 \mathrm{M} \mathrm{pH} \mathrm{7.4)} \mathrm{preparation,}$ were purchased from Merck Life Science (Milan, Italy). Mouse IgG was from Thermo Fisher Scientific (Milan, Italy). Pooled normal human serum was from HyTest Ltd. (Turku, Finland).

\subsection{Methods}

The LPFG was fabricated within a Fibercore PS1250/1500 B/Ge co-doped photosensitive fiber with a $\mathrm{KrF}$ excimer laser (Lambda Physics Compex 110) by using the point-by-point inscription technique. The period of the LPFG was $\Lambda=246 \mu \mathrm{m}$ and the number of grating planes was 123 , with a resulting calculated grating length of $30.012 \mathrm{~mm}$. The period was optimized using theoretical modeling in such a way that the left peak of the dual resonance of the $\mathrm{LP}_{0,2} \mathrm{can}$ be obtained in $\mathrm{C}$ band in water medium after cladding diameter reduction. Detailed calculation is shown in our previous work [24]. The LPFG characterization setup was composed of a SLED source (FiberLabs Inc. SLD1310/1430/1550/1690-10) and an optical spectrum analyzer (OSA; Anritsu MS9030A/9701C). The minimum wavelengths were calculated by fitting the resonant band with a Lorentzian function. The bandwidth was considered from $1300 \mathrm{~nm}$ to $1700 \mathrm{~nm}$ depending on the SLED source band and on the cut-off wavelength of the used fiber (1209 $\mathrm{nm})$.

After inscription, chemical etching of the fiber was done by dipping it in $20 \%$ HF solution; the fiber was kept straight during this process in order to avoid bending induced perturbations caused by the reduced fiber diameter.

For a better handling of the sensor and to eliminate the effect of surrounding temperature fluctuation during the SRI sensitivity measurement and also during immunoassay, the LPFG was inserted inside a thermostated closed flow cell made of poly(methyl methacrylate) (PMMA). Detail of the flow cell and the insertion procedure was described in [24].

SRI sensitivity analysis was performed using $\mathrm{NaCl}$ solutions in water $(0.0-0.5 \% w / v)$ (RI in the range 1.333 to 1.3335 [25]) and the immunoassay was carried out using mouse IgG/Anti mouse IgG interaction [10]. BSA was used to block the nonspecific adsorption. The specificity of the sensor was confirmed using human serum as negative control. Both the SRI sensitivity analysis and the immunoassay were performed inside the flow cell.

\section{Results and Discussion}

The LPFG was fabricated by using the procedure described in Section 2.2. The diameter of the etched LPFG was measured to be $\sim 21 \mu \mathrm{m}$. The spectra of the LPFG after inscription and after cladding diameter reduction is shown in Figure 1. It was clearly observed that after cladding diameter reduction the left peak of $\mathrm{LP}_{0,2} \mathrm{CM}$ was generated within $\mathrm{C}$ band. 


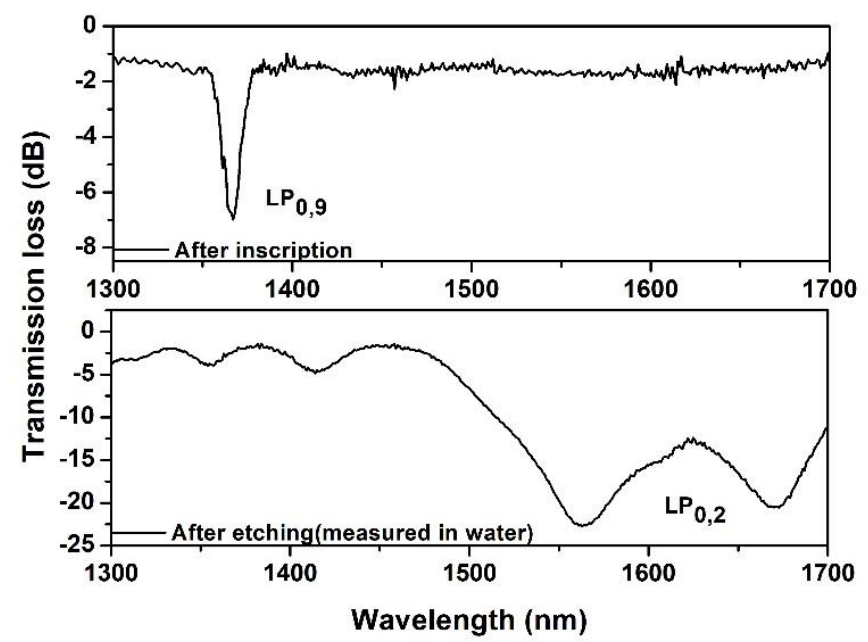

Figure 1. Spectral measurement of LPFG after inscription and after cladding diameter reduction.

The SRI sensitivity analysis was done using the procedure described in Section 2.2 and it was found out to be $8751 \mathrm{~nm} / \mathrm{SRIU}$ as shown in Figure 2.

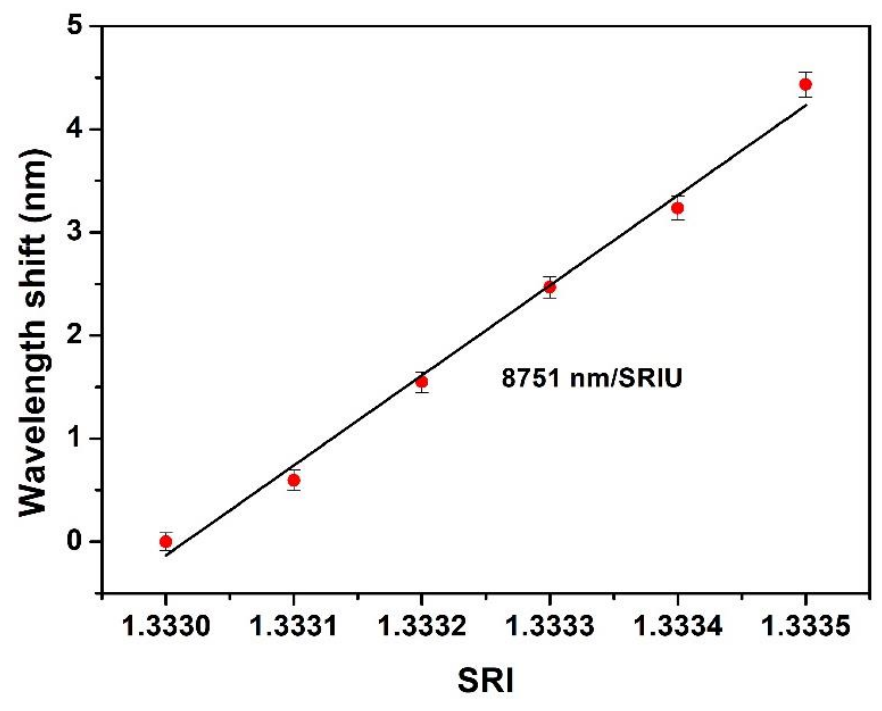

Figure 2. Wavelength shift of dual peak of $\mathrm{LP}_{0,2} \mathrm{CM}$ near TAP as a function of surrounding refractive index.

To perform the immunoassay, mouse IgG $(1000 \mu \mathrm{g} / \mathrm{mL}$ in PBS) was covalently bound on the sensor surface by following the procedure described in [10]. After blocking the non-specific sites on the sensor surface using BSA, a negative control measurement was performed using human serum and then two concentrations of anti-mouse $\operatorname{IgG}(0.0001 \mu \mathrm{g} / \mathrm{mL}$ and $0.001 \mu \mathrm{g} / \mathrm{mL})$ were used to carry out the assay. After each step, rinsing with PBS was performed and also every measurement was done in PBS solution. The dual peak resonant wavelength shift due to the exposure of the sensor to different concentrations of anti-mouse IgG is shown in Figure 3. 


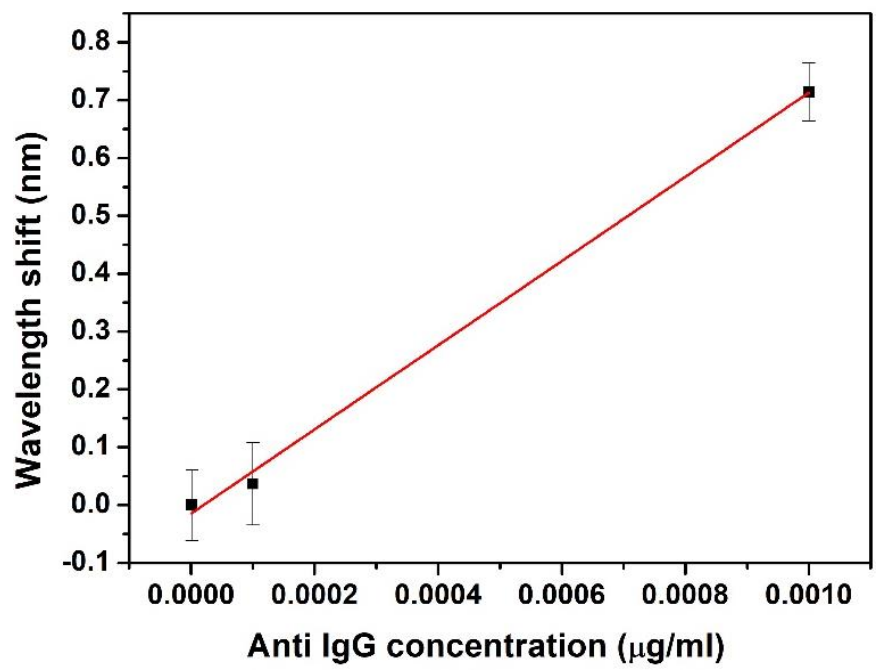

Figure 3. Wavelength shift of dual peak of $\mathrm{LP}_{0,2} \mathrm{CM}$ at different concentration of anti-IgG.

\section{Conclusions}

A reduced cladding diameter LPFG sensor was fabricated with maximum enhancement of evanescent field along with the combine effect of the TAP by working with lowest order of symmetric $\mathrm{CM}\left(\mathrm{LP}_{0,2} \mathrm{CM}\right)$ near TAP. The sensor was characterized by SRI sensitivity and a label free immunoassay was performed using IgG/ Anti IgG interaction in a closed thermostated flow cell.

Acknowledgments: Authors are thankful to SERB, Government of India, for providing overseas visiting doctoral fellowship (ODF/2018/000288) to Tanoy Kumar Dey.

\section{References}

1. Chiavaioli, F.; Baldini, F.; Tombelli, S.; Trono, C.; Giannetti, A. Biosensing with optical fiber gratings. Nanophotonics 2017, 6, 663-679.

2. Korposh, S.; Lee, S.; James, S. Long Period Grating Based Fibre Optic Chemical Sensors. In Fiber Optic Sensors Current Status and Future Possibilities; Smart Sensors, Measurement and Instrumentation Book Series; Springer: 2017; Volume 21, pp. 241-267.

3. James, S.W.; Tatam, R.P. Optical fiber long-period grating sensors: Characteristics and application. Meas. Sci. Technol. 2003, 14, R49-R61.

4. Urrutia, A.; Villar, I.D.; Zubiate, P.; Zamarreño, C.R. A comprehensive review of optical fiber refractometers: Toward a standard comparative criterion. Laser Photonics Rev. 2019, 13, 1900094.

5. Yang, F.; Chang, T.; Liu, T.; Wu, D.; Du, H.; Liang, J.; Tian, F. Label- free detection of Staphylococcus aureus bacteria using long-period fiber grating with functional polyelectrolyte coatings. Biosens. Bioelectron. 2019, $133,147-153$.

6. Esposito, F.; Sansone, L.; Taddei, C.; Campopiano, S.; Giordano, M.; Iadicicco, A. Ultrasensitive biosensor based on long period grating coated with polycarbonate-graphene oxide multilayer. Sens. Actuators B Chem. 2018, 274, 517-526.

7. Janczuk-Richter, M.; Piestrzynska, M.; Burnat, D.; Sezemsky, P.; Stranak, V.; Bock, W.J.; Bogdanowicz, R.; Niedziolka-Jonsson, J.; Smietana, M. Optical investigation of electrochemical process using a long-period fiber grating functionalized by indium tin oxide. Sens. Actuators B Chem. 2019, 279, 223-229.

8. Patrick, H.J.; Kersey, A.D.; Bucholtz, F. Analysis of the response of long period fiber grating to external index of refraction. J. Lightwave Technol. 1998, 16, 1606-1612.

9. Villar, I.D.; Matias, I.R.; Arregui, F.J.; Lalanne, P. Optimization of sensitivity in long period fiber grating with overlay deposition. Opt. Express 2005, 13, 56-69.

10. Chiavaioli, F.; Biswas, P.; Trono, C.; Jana, S.; Bandyopadhyay, S.; Basumallick, N.; Giannetti, A.; Tombelli, S.; Bera, S.; Mallick, A.; et al. Sol-gel-based titania-silica thin film overlay for long period fiber grating based biosensors. Anal. Chem. 2015, 87, 12024-12031. 
11. Pilla, P.; Manzillo, P.F.; Malachovska, V.; Buosciolo, A.; Campopiano, S.; Cutolo, A.; Ambrosio, L.; Giordano, M.; Cusano, A. Long period grating working in transition mode as promising technological platform for label free biosensing. Opt. Exp. 2009, 17, 20039-20050.

12. Pilla, P.; Malachovska, V.; Borriello, A.; Buosciolo, A.; Giordano, M.; Ambrosio, L.; Cutolo, A.; Cusano, A. Transition mode long period grating biosensor with functional multilayer coatings. Opt. Exp. 2011, 19, 512526.

13. Shu, X.; Zhang, L.; Bennion, I. Sensitivity characteristics of long-period fiber grating. J. Lightwave Technol. 2002, 20, 255-266.

14. Biswas, P.; Basumallick, N.; Bandyopadhyay, S.; Dasgupta, K.; Ghosh, A.; Bandyopadhyay, S. Sensitivity enhancement of turn-around-point long period grating by tuning initial coupling condition. IEEE Sens. J. 2015, 15, 1240-1245.

15. Chiang, K.S.; Liu, Y.; Ng, M.N.; Dong, X. Analysis of etched long-period fiber grating and its response to external refractive index. Electron. Lett. 2000, 36, 966-967.

16. Śmietana, M.; Koba, M.; Mikulic, P.; Bock, W.J. Measurements of reactive ion etching process effect using long-period fiber gratings. Opt. Exp. 2014, 22, 5986-5994.

17. Zou, F.; Liu, Y.; Mou, C.; Zhu, S. Optimization of refractive index sensitivity in nano-film coated longperiod fiber grating near dispersion turning point. J. Lightwave Technol. 2019, doi:10.1109/JLT.2019.2949373.

18. Villar, I.D.; Cruz, J.L.; Socorro, A.B.; Corres, J.M.; Matias, I.R. Sensitivity optimization with cladding-etched long period fiber gratings at dispersion turning point. Opt. Exp. 2016, 24, 17680-17685.

19. Villar, I.D. Ultrahigh-sensitivity sensors based on thin-film coated long period grating with reduced diameter, in transition mode and near the dispersion turning point. Opt. Exp. 2015, 23, 8389-8398.

20. Piestrzyńska, M.; Dominik, M.; Kosiel, K.; Janczuk-Richter, M.; Szot-Karpińska, K.; Brzozowska, E.; Shao, L.; Niedziółka-Jonsson, J.; Bock, W.J.; Śmietana, M. Ultrasensitive tantalum oxide nano-coated long-period grating for detection of various biological targets. Biosens. Bioelectron. 2019, 133, 8-15.

21. Śmietana, M.; Koba, M.; Mikulic, P.; Bock, W.J. Towards refractive index sensitivity of long period gratings at level of tens of $\mu \mathrm{m}$ per refractive index unit: Fiber cladding etching and nano-coating deposition. Opt. Exp. 2016, 24, 11897-11904.

22. Smietana, M.; Koba, M.; Mikulic, P.; Bock, W.J. Combined plasma-based fiber etching and diamond-like carbon nanooverlay deposition for enhancing sensitivity of long-period grating. J. Lightwave Technol. 2016 34, 4615-4619.

23. Dey, T.K.; Biswas, P.; Bandyopadhyay, S.; Basumallick, N.; Bandyopadhyay, K.D.S. Sensitivity analysis of a dispersed clad mode to surrounding refractive index. In Proceedings of the 2015 International Conference on Microwave and Photonics (ICMAP), Dhanbad, India, 11-13 December 2015; doi:10.1109/ICMAP.2015.7408709.

24. Tombelli, T.K.D.S.; Biswas, P.; Giannetti, A.; Basumallick, N.; Baldini, F.; Bandyopadhyay, S.; Trono, C. Analysis of the Lowest Order Cladding Mode of Long Period Fiber Gratings near Turn Around Point. J. Lightwave Technol. 2020, doi:10.1109/JLT.2020.2987795.

25. Barbosa, E.A.; Silva, D.M.; Preto, A.O.; Verzini, R. Design, construction, and performance of a real-time holographic refractometry prototype for liquid analysis. Rev. Sci. Instrum. 2011, 82, 013103.

Publisher's Note: MDPI stays neutral with regard to jurisdictional claims in published maps and institutional affiliations.

(C) 2020 by the authors. Submitted for possible open access publication under the terms and conditions of the Creative Commons Attribution (CC BY) license (http://creativecommons.org/licenses/by/4.0/). 